\title{
Callous-unemotional Traits, moralische Identität und Bullying im Jugendalter
}

\author{
Neele Schipper und Ute Koglin \\ Institut für Sonder- und Rehabilitationspädagogik, Carl von Ossietzky Universität Oldenburg
}

\begin{abstract}
Zusammenfassung: Theoretischer Hintergrund: Die Rolle der Moral im Kontext pro- und antisozialer Verhaltensweisen wird sowohl national als auch international diskutiert. Im Zusammenhang mit antisozialen Verhaltensweisen von Jugendlichen werden zudem vermehrt Callousunemotional Traits betrachtet. Fragestellung: Die vorliegende Studie untersucht den Zusammenhang zwischen CU-Traits, der moralischen Identität und Bullying. Methode: Insgesamt bearbeiteten $N=749$ Jugendliche einen Fragebogenkatalog. SPSS und AMOS wurden zur Analyse der vorliegenden Daten genutzt. Ergebnisse: Die Ergebnisse zeigen direkte Effekte von Callousness, Uncaring und der moralischen Identität auf Bullying. Die Skalen Uncaring und Callousness zeigen einen Zusammenhang mit moralischer Identität und Bullying. Diskussion und Schlussfolgerung: Die Ergebnisse deuten an, dass die moralische Identität und die damit einhergehende moralische Entwicklung wichtige Prädiktoren für das Verhalten von Jugendlichen sind.
\end{abstract}

Schlüsselwörter: Bullying, Callous-unemotional Traits, moralische Identität, Jugendalter

Callous-Unemotional Traits, Moral Identity, and Bullying Perpetration in Adolescents

Abstract: Theoretical Background: The influence of moral identity on prosocial and antisocial behavior in adolescents has been highlighted in numerous studies. However, even if moral principles are central to the self, they do not always directly and consistently lead to moral behavior. Callous-unemotional (CU) traits have been associated with bullying among adolescents. However, at this point we do not know whether the association of each of the three sub-constructs of CU-traits are associated with moral identity. Objectives: The aim of the present study was to examine whether CU traits and moral identity were related to moral decisions. Further, the aim of the current study was to investigate the association between $\mathrm{CU}$ traits and bullying perpetration, and to determine whether these associations were mediated by moral identity. Method: Cross-sectional data was collected in 2020 . A total of 749 adolescents $\left(46.5 \%\right.$ boys; $\left.M_{\text {Age }}=14.14, S D_{\text {Age }}=1.85\right)$ from different schools in Germany completed an online questionnaire. Questions regarding moral identity (Koglin, 2017), callous-unemotional traits (Essau, Sasagawa, \& Frick, 2006), and bullying (KFN, Bergmann et al., 2017) were answered by the participants. SPSS Statistics and SPSS AMOS were used to analyze the collected data. Path analysis was used to test the hypotheses. Results: The results of this study contribute to the description of the explanatory processes for the connection between CU traits, moral identity, and bullying, for a national sample. The hypothesized path model demonstrated good fit values $(N=749 ; \chi 2 / d f=.032, p=.858, C F I=1.00, N F I=1.00, T L I=1.02$, RMSEA $=.000$ ). Results showed that callousness and uncaring were negatively related to moral identity and positively related to bullying perpetration. Moral identity was negatively associated with bullying perpetration. Mediation analysis showed that moral identity was a significant mediator between CU-traits and bullying perpetration. The findings indicate that moral identity plays an essential role in bullying perpetration in adolescents and that callousness and uncaring appears to be the most important CU-traits in relation to moral identity and bullying perpetration. Discussion and Conclusion: The results suggest that moral identity and the associated moral development are important predictors of adolescents' behavior. Furthermore, these findings point out that moral identity plays an essential role in bullying perpetration in adolescents. The present results confirm that the promotion of social-emotional competencies can be used to prevent bullying during adolescence. To reduce $\mathrm{CU}$ traits, interventions that start very early would have to be used to promote social-emotional development. Further research should explore this interplay in order to gain a holistic and comprehensive understanding of the underlying mechanisms.

Keywords: bullying, callous-unemotional traits, moral identity, adolescents

Die Schule nimmt einen wesentlichen Bestandteil im Alltag von Kindern und Jugendlichen ein. Dieser Kontext bietet einen Raum für Konflikte unter Gleichaltrigen, die sich beispielsweise im Bullying äußern. Eine Studie von Craig et al. (2009) untersuchte jugendliche Schulkinder aus 40 Ländern. Die Prävalenzraten zeigten, dass 10.7\% der Jugendlichen Täter_innen, $12.6 \%$ Opfer und 3.6\% Täter_innen/Opfer wurden. Die Folgen von Bullying erweisen sich als weitreichend. In der Literatur werden enge Zusammenhänge zwischen Viktimisierung und emotionalen Problemen wie Depression und Suizidalität berichtet (Klomek, Barzilay \& Apter, 2019; Olweus, 2013). 
Analysen ergaben, dass Bullying die Chancen für emotionale Probleme um das 2.4fache, für Suizidalität um das 2.8fache und für selbstverletzendes Verhalten um das 3.Ofache erhöhte (Jantzer, Haffner, Parzer \& Resch, 2012). Die Leittragenden dieser Verhaltensweisen sind jedoch nicht nur die Opfer, auch die Täter_innen selbst leiden unter den Folgen. Es zeigen sich negative Entwicklungsverläufe sowie ein höheres Risiko für soziale Ausgrenzung (Petermann \& Koglin, 2015). Die Rolle der Moral im Kontext pro- und antisozialer Verhaltensweisen wird sowohl national als auch international diskutiert (Malti, Gummerum, Keller \& Buchmann, 2009; Malti \& Krettenauer, 2013; Muratori et al., 2017). Im Zusammenhang mit antisozialen Verhaltensweisen von Jugendlichen werden zudem vermehrt Callous-unemotional Traits (CU-Traits), zu Deutsch auch emotionale Unbeteiligtheit, betrachtet, die sich durch mangelnde Reue und Empathie kenntlich machen (Koglin \& Petermann, 2012).

Insgesamt deutet die Literatur darauf hin, dass Kinder und Jugendliche, bei denen ein höheres Risiko besteht, störende Verhaltensweisen zu zeigen, auch auf individueller Ebene Schwierigkeiten aufweisen. Diese zeigen sich beispielweise in moralischen Emotionen (z. B. mangelndes Einfühlungsvermögen und mangelndes Schuldgefühl) oder auch in moralischen Kognitionen (z.B. schwaches moralisches Urteilsvermögen und geringe persönliche Verantwortung). Ziel der vorliegenden Studie ist es $\mathrm{zu}$ überprüfen, ob CU-Traits und die moralische Identität Risikofaktoren für Bullying in einer Stichprobe von Jugendlichen darstellen.

\section{Theoretischer Hintergrund}

Olweus (1993) definiert Bullying als wiederholte und systematisch schädigende Handlungen, denen ein_e Schüler_in direkt oder indirekt, durch einen Schüler/eine Schülerin oder mehrere Schüler_innen, ausgesetzt ist. Dabei besteht ein psychisches und physisches Ungleichgewicht zwischen Täter_in und Opfer (Scheithauer, Hayer \& Petermann, 2003). Es lassen sich drei wesentliche Merkmale von Bullying erkennen: eine schädigende Intention des Täters/der Täterin, die Wiederholung dieses Verhaltens und ein Macht-Ungleichgewicht zwischen Opfer und Täter_in. Auf der Verhaltensebene lassen sich drei Formen von Bullying unterscheiden: physisches Bullying (körperliche Übergriffe), verbales Bullying (verspotten, beleidigen, beschimpfen) und relationales Bullying (intendierte Schädigung einer Person durch sozialen Ausschluss, etwa durch Ausgrenzungen oder Verleumdungen) (Scheithauer et al., 2003). Marées und Petermann (2009) weisen darauf hin, dass Jungen und Mädchen et- wa gleich häufig in den Rollen Täter_in, Opfer oder Täter_in - Opfer zu finden sind. Das kriminologische Forschungsinstitut (KFN) berichtet, dass $45.9 \%$ der Jugendlichen schon einmal gehänselt, ausgeschlossen oder nicht beachtet worden sind (Bergmann, Baier, Rehbein \& Mößle, 2017). Nach Olweus (1993, 2013) zeichnen sich Gewalttäter und Gewalttäterinnen im schulischen Kontext durch körperliche und sportliche Überlegenheit, eine starke Ausprägung des Machtmotivs, Impulsivität und eine geringe Frustrationstoleranz, ein starkes Misstrauen und Widerstand gegen Erwachsene, wenig Mitleid, ein starkes Selbstwertgefühl und gesellschaftsfeindliches Verhalten aus. Diese Merkmale zeigen Parallelen zu den Callous-unemotional-Traits (CU-Traits), wie aggressives Verhalten, geringe Empathie und manipulierendes Verhalten (American Psychiatric Association, 2013; Olweus 1993, 2013; Koglin \& Petermann, 2012).

Callous-unemotional Traits werden herangezogen, um eine besonders schwerwiegende Form aggressiv-dissozialen Verhaltens im Kindes- und Jugendalter $\mathrm{zu}$ beschreiben. Sie werden über eine geringe Empathie, Reue und Prosozialität definiert und gehen zurück auf das Konstrukt der Psychopathie im Erwachsenenalter (Hare, 2003; Koglin \& Petermann, 2007). Weitere Merkmale sind Sorglosigkeit bei schlechten Leistungen in der Schule, bei der Arbeit oder weiteren Lebensbereichen und ein oberflächiger oder manipulativer Emotionsausdruck (Hare, 1999; Koglin \& Petermann, 2007). Nach Frick (2004) beziehen sich CU-Traits auf die Dimensionen Callousness, Uncaring sowie Unemotional. Callousness umfasst gefühllose bzw. kaltherzige Einstellungen gegenüber anderen Personen, was sich in einem Mangel an Empathie, Schuldgefühlen und Reue für Vergehen äußert. Uncaring erfasst Gleichgültigkeit gegenüber den eigenen Leistungen bei der die Bewältigung von Aufgaben oder den Gefühlen anderer Personen. Unemotional umfasst den fehlenden Emotionsausdruck (Essau, Sasagawa \& Frick, 2006). CU-Traits erweisen sich als Prädiktor für Verhaltensprobleme (Koglin \& Petermann, 2012). Frick und Salekin (2005) betonen, dass CU-Traits besonders bei Kindern mit Störungen des Sozialverhaltens maßgeblich zur Entwicklung schwerer Formen aggressiv-dissozialen Verhaltens führen. Welche Wirkmechanismen diesem Zusammenhang unterliegen, ist bislang noch ungeklärt. Bisherige Studien verdeutlichen jedoch, dass das Vorhandensein von CU-Traits durch eine besonders negative Entwicklungsdynamik gekennzeichnet ist (Frick \& Salekin, 2005).

In der Forschung zur moralischen Identitätsentwicklung werden Ansätze der Persönlichkeitsforschung herangezogen, die erklären sollen, warum Individuen die eigenen moralischen Forderungen einhalten. Das Konzept der moralischen Identität geht auf Blasi (1983) zurück, der 
die Theorie insbesondere durch sein Self-Model, welches auf die Verbindung zwischem moralischem Urteilsvermögen und moralischem Handeln eingeht, prägt. Die Forschung im Gebiet der moralischen Identität befasst sich mit den Gründen der Einhaltung von eigenen und externen moralischen Forderungen. In der Literatur wird zur moralischen Identität synonym auch der Begriff des moralischen Selbst (moral self) verwendet (Krettenauer, 2013). Nach Blasi (1983) gilt die moralische Identität als wesentlicher Einflussfaktor der Motivation und der Handlung. Dies bedeutet, dass eigene moralische Überzeugungen als unabdingbarer Aspekt der Identitätsbildung gelten, genauer muss es dem Individuum etwas bedeuten, moralisch $\mathrm{zu}$ handeln. Handlungen, die einen Verstoß gegen ein moralisches Prinzip bedeuten, versteht Blasi (1983) als Betrug des eigenen Selbstkonzeptes. Sind moralische Werte stark in das Selbst integriert, ist ein Individuum aufgrund der Selbstkonsistenz motivierter, moralisch zu handeln (Doering, 2013). Nach Hardy und Carlo (2005) ist das moralische Selbst für moralisches Handeln verantwortlich. Aktuelle Studien stützen die Annahme von Blasi (1983) und knüpfen an diese an und definieren die moralische Identität als das Ausmaß, in dem es der Person wichtig ist, eine moralische Person zu sein (Hardy \& Carlo, 2011). Dieses Bedürfnis kann individuell variieren und entweder stärker oder schwächer ausgeprägt sein. Diese Auffassung versteht die moralische Identität als Teil des Selbstkonzeptes, welches sich inmitten einer Reihe von verschiedenen Eigenschaften organisiert (Aquino \& Reed, 2002). Die Definition von Aquino und Reed (2002) weist viele Parallelen zu der von Hardy und Carlo (2011) auf, berücksichtigt zusätzlich aber noch Theorien des Selbstverständnisses und der sozialen Identität.

\section{Vorliegende Studie}

Das Ziel dieser Studie ist es, die Rolle der moralischen Identität und der CU-Traits als Prädiktoren für Bullying $\mathrm{zu}$ identifizieren und die Rolle der moralischen Identität als Mediator für den Einfluss von CU-Traits auf Bullying zu untersuchen. Aus aktuellen Forschungsarbeiten lässt sich entnehmen, dass CU-Traits zunehmend in Verbindung mit Bullying (Thornberg \& Jungert, 2017; Zych, Ttofi \& Farrington, 2019) gebracht werden und als bedeutsame Prädiktoren gelten (Munoz, Qualter \& Padgett, 2011). Weiterhin wird Bullying zunehmend als ein von der Moral ausgehendes Problem diskutiert (Gini, Pozzoli \& Bussey, 2015; Grundherr, Geisler, Stoiber \& Schäfer, 2017; Hymel, Rocke Henderson \& Bonanno 2005; Malti \& Krettenauer, 2013; Thornberg \& Jungert, 2016; Wang, Ryoo, Swearer,
Turner, Goldberg, 2017). Bullying und andere Formen aggressiv-dissozialen Verhaltens sind nicht mit moralischen Vorstellungen und einer positiv ausgeprägten moralischen Identität vereinbar. Aufgrund der bisherigen Forschungsergebnisse stellt sich die Frage nach dem $\mathrm{Zu}$ sammenspiel der CU-Traits, der moralischen Identität und Bullying. Beruhend auf den dargestellten Annahmen und empirischen Befunden soll die Rolle der moralischen Identität als Mediator in der Beziehung von CU-Traits auf Bullying mittels Pfadanalyse geprüft werden. Es wird vermutet, dass sich ein negativer indirekter Einfluss der CUTraits auf Bullying zeigt, der über die moralische Identität vermittelt wird. Weiterhin wird vermutet, dass ein positiver, direkter Zusammenhang für den Einfluss der CUTraits auf Bullying, sowie ein negativer Zusammenhang für den Einfluss von moralischer Identität auf Bullying besteht.

\section{Methode}

\section{Stichprobe und Durchführung}

Bei der vorliegenden Studie handelt es sich um eine Querschnittsstudie. Die Datenerhebung erfolgte im Frühjahr/Sommer 2020 an Regelschulen aller Schulformen in Niedersachsen der Klassenstufen 5-13. Es liegt ein positives Votum der Kommission für Forschungsfolgenabschätzung und Ethik der Universität sowie der Stabsstelle für Datenschutz- und Informationssicherheitsmanagement vor. Zusätzlich wurde die Zustimmung durch die zuständige Landesschulbehörde sowie durch die Schulleitungen eingeholt. Die Jugendlichen und die Eltern der Jugendlichen wurden über ein Informationsschreiben und eine dazugehörige Einwilligungserklärung um ihr Einverständnis zur Teilnahme gebeten. Die Teilnahme am Projekt war freiwillig. Weiterhin wurden die Jugendlichen und ihre Eltern darüber aufgeklärt, dass die Antworten nur in anonymisierter Form weiterverarbeitet werden und die Bearbeitung des Fragebogens jederzeit abgebrochen werden kann. Die Beantwortung des Fragebogens erfolgte mittels Online-Fragebogen.

Insgesamt bearbeiteten $N=749$ Jugendliche $\left(M_{\text {Alter }}=\right.$ 14.14, $\left.S D_{\text {Alter }}=1.85\right)$ den Fragebogenkatalog, davon $n=$ 348 (46.5\%) männliche Jugendliche. Es handelt sich um eine ad-hoc Stichprobe, bei der $9.4 \%$ der Jugendlichen angaben, in einem anderen Land als Deutschland geboren zu sein. Diese Information ergab sich aus der Angabe zur gesprochenen Muttersprache und zum Geburtsland der Jugendlichen. Es wurden keine spezifischen Informationen zu einem Migrationshintergrund durch die Angaben des Geburtslandes der Eltern abgefragt (nach Defini- 
tion des Statistischen Bundesamtes, 2013), sodass der Migrationshintergrund wahrscheinlich unterschätzt wird. $35.8 \%$ der Jugendlichen besuchen das Gymnasium, $44.1 \%$ eine Realschule, $2.4 \%$ eine Hauptschule, $16.3 \%$ eine Oberschule und $1.4 \%$ gaben an, eine Gesamtschule zu besuchen.

\section{Erhebungsinstrumente}

Die Erhebung fand anhand eines Online-Fragebogens statt. Dieser setzt sich aus Fragen zum Bullying (KFN; Bergmann et al., 2017), zur moralischen Identität (Koglin, 2017) und zu den Callouss-unemotional Traits (ICU; Essau et al., 2006; Frick, 2004) zusammen.

Anhand von Items des KFN (Kriminologisches Forschungsinstitut Niedersachsen) wird Bullying erfasst (Bergmann et al., 2017). Die Antworten sollten sich auf das letzte Schulhalbjahr beziehen und konnten jeweils von nie (1) bis mehrmals (6) beantwortet werden (Bergmann et al., 2017). Unter Bullying wurden verschiedene Gewaltdelikte aus Täterperspektive erfragt z. B. „Ich habe einen anderen Schüler absichtlich geschlagen oder getreten." ( $\alpha=.79 ; 10$ Items).

Zur Erfassung der moralischen Identität entwickelte Koglin (2017) insgesamt 18 Items. Die Skala fordert die Schülerinnen und Schüler dazu auf, die subjektive Wichtigkeit 18 menschlicher Eigenschaften zu bewerten (z. B. „selbstlos“, „mitfühlend“). Neun der Items erfassen die moralische Identität, die anderen Items dienen als Distraktoren. Die Bewertung erfolgt auf einer vierstufigen, unipolaren Ratingskala von nicht wichtig für mich (1) bis sehr wichtig für mich (4) ( $\alpha=.78$; 9 Items).

Das Inventory of Callous-unemotional Traits (ICU; Essau et al., 2006; Frick, 2004) bildet die emotionale Unbeteiligtheit ab. Das ICU erfasst CU-Traits, wie fehlende Reue oder Schuld, Gleichgültigkeit, das Fehlen von Empathie sowie Sorglosigkeit gegenüber eigenen Leistungen (Petermann \& Koglin, 2013). Bewertet werden 24 Aussagen anhand einer vierstufigen, bipolaren Skala von stimmt gar nicht (1) bis stimmt genau (4). Grundlage des ICU ist eine Skala aus dem Antisocial Process Screening Device von Frick und Hare (2001). Das ICU besteht aus drei Skalen: Callousness z.B. „Es ist mir egal, wen ich verletzte, um zu bekommen, was ich will." $(\alpha=.69$; 9 Items), Unemotional z. B. „Ich verstecke meine Gefühle vor Anderen.“ ( $\alpha=.72 ; 5$ Items), Uncaring (rekodiert) „Ich fühle mich schlecht oder schuldig, wenn ich etwas falsch gemacht habe." ( $\alpha=77 ; 8$ Items) (Essau et al., 2006).

\section{Auswertungsstrategie}

SPSS und AMOS wurden zur Analyse der vorliegenden Daten genutzt. Das Signifikanzniveau wurde auf $\alpha=.05$ gesetzt; alle Überprüfungen erfolgten zweiseitig. Die MCAR (Missing Completely at Random; Little, 1988) Bedingung ist in den verwendeten Daten erfüllt $\left(\chi^{2}=25.59\right.$, $D F=26, p=.49)$ und erlaubt die Full Information Maximum Likelihood Schätzung des Regressionskoeffizienten in der Datenanalyse (Baltes-Götz, 2008). Vor der Analyse wurden alle Skalen z-transformiert, um uneinheitliche Skalierungen auf einen gleichen Maßstab zu setzen und die Stichprobenwerte vergleichbar zu machen. Für alle Pfade im Modell wurden vorab Korrelationen ermittelt. Die Voraussetzungen für die vorgenommenen Analysen der Daten wurden geprüft. IBM SPSS AMOS 25 wurde genutzt, um die Rolle der moralischen Identität als Mediator in der Beziehung von den Subskalen der ICU als unabhängige Variablen (UV's) auf Bullying als abhängige Variable (AV) zu überprüfen. Im Rahmen des Pfadmodells wurden sowohl die direkten Effekte zwischen den Variablen als auch die indirekten Mediationseffekte bestimmt. Die Auswirkungen der Kontrollvariablen wurden ebenfalls analysiert. Diese Analyse erfolgte zunächst mittels Nested Model Comparison, die zeigte, dass das umfangreichere Modell mit den Kontrollvariablen Alter und Geschlecht besser zu den Daten passt. Die Pfadanalyse wurde mit der Bootstrap-Methode mit Vertrauensschätzungen durchgeführt. In der vorliegenden Studie wurden Konfidenzintervalle auf einem $95 \%$-Niveau für indirekte Effekte mit einem Bootstrap von 1000 Stichproben ermittelt. Zur Einschätzung der Modellgüte wurden die Gütemaße $\chi 2 / d f$, RMSEA, TLI, CFI und NFI herangezogen. Für $\chi 2 / d f$ gilt ein guter Modellfit $\leq 3$ (Homburg \& Giering, 1996). Der Root Mean Square Error of Approximation (RMSEA) ist ein Maß der durchschnittlichen Diskrepanz pro Freiheitsgrad. Hier bestätigen Fit-Werte unter 0.8 das berechnete Modell (Browne \& Cudeck, 1993). Der CFI-Wert (Comparative Fit Index), NFI-Wert (Normed Fit Index) und der TLI-Wert (Tucker-Lewis-Index) sollten sich zeitgleich über dem Wert 0.9 bewegen, um eine gute allgemeine Modellgüte zu bestätigen (Arbuckle, 2012; Homburg \& Baumgartner, 1995).

\section{Ergebnisse}

Die deskriptiven Statistiken sind für alle Variablen, die in das Pfadmodell aufgenommen wurden, in Tabelle 1 dargestellt. Die Interkorrelationen der Variablen sind in Tabelle 2 aufgeführt. Zwischen denen im Modell aufgenommen unabhängigen Variablen (ICU Skalen) und der Me- 
diatorvariable moralische Identität sowie der abhängigen Variable Bullying bestehen durchweg signifikante $\mathrm{Zu}-$ sammenhänge.

Tabelle 1. Deskriptive Statistik für alle Variablen im Pfadmodell ( $N=$ 749)

\begin{tabular}{lcccc}
\hline & $M$ & SD & \multicolumn{2}{c}{ Range } \\
& & & Min & Max \\
\hline Unemotional & 12.54 & 3.12 & 5.00 & 20.00 \\
Uncaring & 15.24 & 4.10 & 8.00 & 32.00 \\
Callousness & 13.97 & 3.85 & 9.00 & 33.00 \\
Moralische Identität & 30.35 & 3.59 & 9.00 & 36.00 \\
Bullying & 13.26 & 4.40 & 10.00 & 60.00 \\
\hline
\end{tabular}

Anmerkungen: $M=$ Mittelwert; $S D=$ Standardabweichung.

Tabelle 2. Interkorrelationen zwischen den Variablen des Pfadmodells

\begin{tabular}{|c|c|c|c|c|c|}
\hline & 1 & 2 & 3 & 4 & 5 \\
\hline Unemotional & 1 & & & & \\
\hline Uncaring & $.26^{\star \star}$ & 1 & & & \\
\hline Callousness & $.31 * *$ & $.48 * *$ & 1 & & \\
\hline Moralische Identität & $-.21 * \star$ & $-.49 * *$ & $-.38 * \star$ & 1 & \\
\hline Bullying & $.13^{\star \star}$ & $.32 \star \star$ & $.41 \star \star$ & $-.33 * \star$ & 1 \\
\hline
\end{tabular}

In Abbildung 1 ist das prognostizierte Pfadmodell mit den direkten Effekten der Pfadkoeffizienten illustriert. Zur Überprüfung des Einflusses der Callous-unemotional Traits auf die moralische Identität und auf Bullying sowie des Einflusses der moralischen Identität auf Bullying, wurden diese pfadanalytisch untersucht. Weiterhin wurden das Alter und das Geschlecht kontrolliert.

Zwei der drei Subskalen der ICU stehen in einem direkten Zusammenhang zur moralischen Identität. Es zeigen sich signifikante, direkte Effekte von Uncaring auf die moralische Identität $(\beta=-.46, p<.001)$ und Callousness auf die moralische Identität $(\beta=-.18, p<.001)$. Es besteht eine direkte Beziehung von Uncaring auf Bullying $(\beta=.12$, $\mathrm{p}<.01)$, Callousness auf Bullying $(\beta=.33, p<.001)$ sowie von der moralischen Identität auf Bullying ( $\beta=-.20, p<$ .001). Das Geschlecht der Jugendlichen hat einen signifikanten Effekt auf die Variablen Callousness ( $\beta=-.23, p<$ $.001)$, Uncaring $(\beta=.25, p<.001)$, die moralische Identität $(\beta=-.07, p<.05)$ und Bullying $(\beta=-.07, p<.05)$. Das Alter der Jugendlichen hat einen signifikanten Effekt auf die Variablen Callousness $(\beta=.09, p<.05)$, Unemotional $(\beta=.08 p<.05)$, die moralische Identität $(\beta=-.08, p<$ $.05)$ und Bullying $(\beta=.09, p<.01)$. Die indirekten Mediationseffekte sind in Tabelle 3 dargestellt. Für alle pos- tulierten abhängigen Variablen lassen sich signifikante, indirekte Mediationseffekte berichten.

Für das Modell ergibt sich eine Gesamtvarianzaufklärung von $28 \%$ für Bullying und eine Varianzaufklärung von $40 \%$ für die moralische Identität unter einem angemessenen Modell-Fit $(N=749 ; \chi 2 / d f=.032, p=.858, C F I$ $=1.00, N F I=1.00, T L I=1.02$, RMSEA $=.000)$.

Tabelle 3. Indirekte Effekte im Pfadmodell

\begin{tabular}{|c|c|c|c|c|}
\hline & \multirow[b]{2}{*}{$\begin{array}{c}\text { Pfadkoeffizient } \\
\beta\end{array}$} & \multirow[b]{2}{*}{$\begin{array}{l}\text { Bootstrap } \\
\quad S D\end{array}$} & \multicolumn{2}{|c|}{$\begin{array}{c}95 \% \text { Konfidenz- } \\
\text { intervall }\end{array}$} \\
\hline & & & $\begin{array}{l}\text { untere } \\
\text { Grenze }\end{array}$ & $\begin{array}{l}\text { obere } \\
\text { Grenze }\end{array}$ \\
\hline \multicolumn{5}{|c|}{ Bullying $($ AV $) \leftarrow$} \\
\hline \multicolumn{5}{|l|}{$\begin{array}{l}\text { Moralische } \\
\text { Identität }\end{array}$} \\
\hline Callousness & $.040 * *$ & .016 & .011 & .077 \\
\hline Uncaring & $.092 * \star$ & .031 & .036 & .155 \\
\hline Unemotional & $.020 * *$ & .008 & .003 & .038 \\
\hline \multicolumn{5}{|c|}{ Anmerkungen: ${ }^{*} p<.05 ; * \star p<.01 ; * \star * x<.001$} \\
\hline
\end{tabular}

Das Ziel dieser Studie war es, die moralische Identität und die CU-Traits als Prädiktoren für Bullying sowie die Rolle der moralischen Identität als Mediator für den Einfluss von CU-Traits auf Bullying zu überprüfen. Die Ergebnisse zeigen, dass hohe Ausprägungen auf den Skalen Callousness und Uncaring mit Bullying einhergehen. Dieser $\mathrm{Zu}$ sammenhang wird über die moralische Identität der Jugendlichen mediiert. Es zeigen sich direkte Effekte von Uncaring, Unemotional und Callousness auf die moralische Identität. Dieses Ergebnis entspricht der Annahme von Hart, Atkins und Ford (1998) zur Bedeutung von personengebundenen Eigenschaften für die moralische Identität. Hart (2005) geht davon aus, dass die Entwicklung der moralischen Identität von Jugendlichen aus der Persönlichkeit hervorgeht. Im Hinblick auf Uncaring fiel der Pfadkoeffizient zur moralischen Identität größer aus als die Pfadkoeffizienten der anderen beiden Dimensionen. Dies bedeutet, je weniger ein Jugendlicher/eine Jugendliche die Belange und Gefühle anderer als gleichgültig betrachtet, desto wichtiger ist es dem Jugendlichen/ der Jugendlichen, eine moralische Person zu sein. Es zeigen sich direkte Effekte von Uncaring und Callousness auf Bullying. Diese Zusammenhänge stützen die Ergebnisse aktueller Studien, die insbesondere die Dimesionen Uncaring und Callousness im Zusammenhang mit Bullying hervorbrachten (z.B. Kurek, Jose \& Stuart, 2019; Thornberg \& Jungert, 2017; Wright, Harper \& Wachs, 


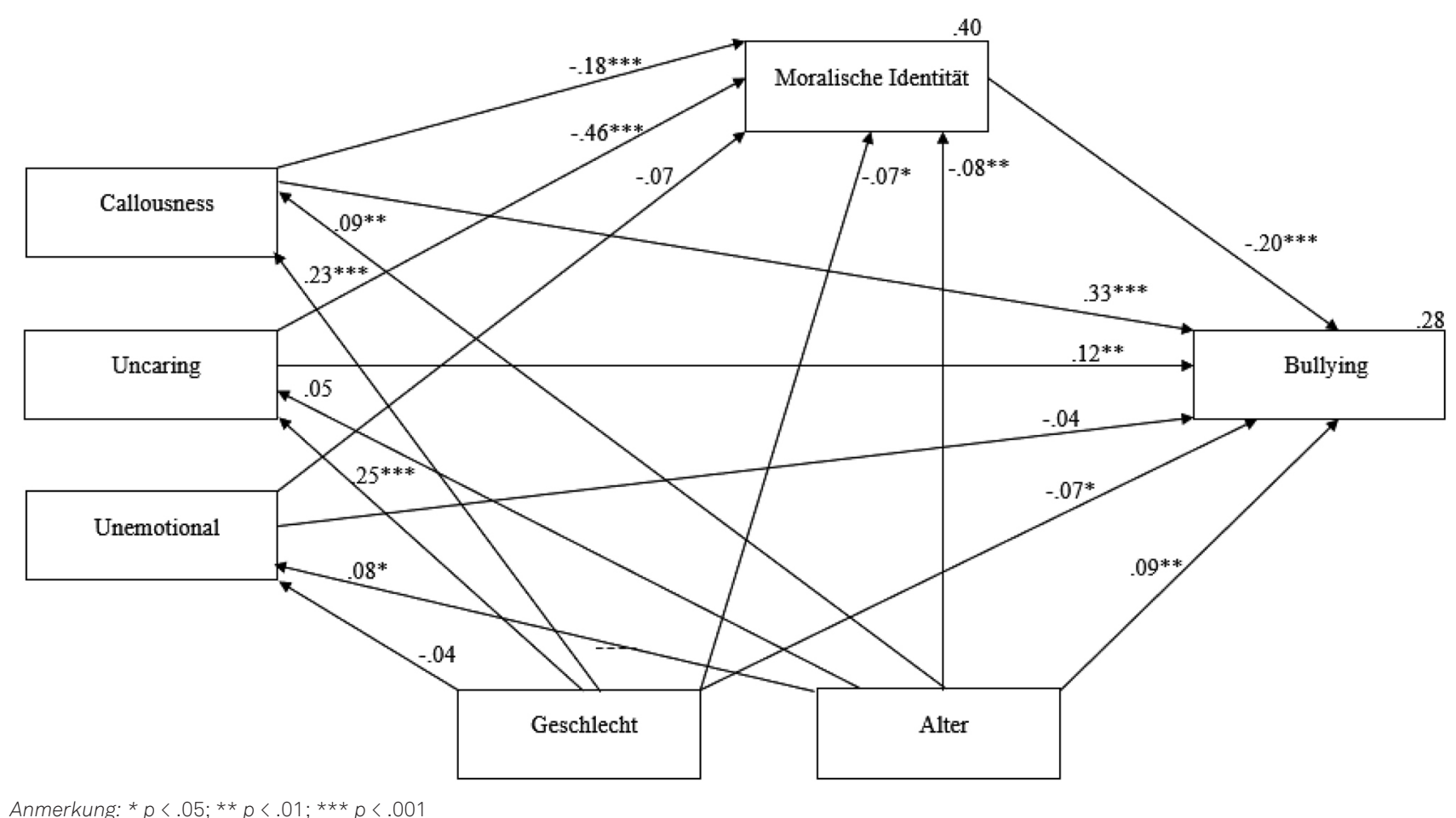

Abbildung 1. Pfadmodell mit direkten Effekten der abhängigen und unabhängigen Variablen.

2019; Zych et al., 2019). In der vorliegenden Studie erwies sich Unemotional hingegen nicht als Prädiktor für Bullying. Nach Cardinale und Marsh (2020) könnte das durch eine unzureichende psychometrische Qualität dieser Skala erklärt werden. Sie berichten aus einer Meta-Analyse, dass nur für die anderen beiden Skalen eine gute interne Konsistenz und externale Validität ermittelt werden konnte, aber nicht für die Skala Unemotional. Andererseits liegt die interne Konsistenz in der vorliegenden Studie bei .72. Munoz et al. (2011) weisen inhaltlich darauf hin, dass Kinder, die eine gleichgültige Haltung gegenüber den Gefühlen und Werten anderer Menschen haben, eher zu direkten Formen von Bullying neigen. Emotionen haben dabei nach Munoz et al. (2011) keinen Einfluss auf Bullying, wenn es einem ohnehin egal ist, Gleichaltrigen zu schaden. Von allen drei Subskalen der CU-Traits zeigt sich der stärkste Effekt von Callousness auf Bullying. Dies bedeutet, dass Jugendliche durch ihre Kaltherzigkeit und dem Mangel an Bedenken hinsichtlich des Wohlergehens anderer, Schaden und Leid zufügen und somit zum Täter/ zur Täterin werden.

Die moderaten Zusammenhänge von CU-Traits und Bullying in dieser Studie decken sich mit aktuellen Forschungsarbeiten (Ciucci, Baroncelli, Franchi, Golmaryami \& Frick, 2014; Fanti, Frick \& Georgiou, 2009; Munoz et al., 2011; Thornberg \& Jungert, 2017). Darüber hinaus kann gezeigt werden, dass der Einfluss von Uncaring auf Bullying, Callousness auf Bullying und Unemotional auf
Bullying durch die moralische Identität mediiert wird. Zudem besteht ein direkter Effekt der moralischen Identität auf Bullying. Die Ergebnisse lassen sich der Annahme von Blasi (1983) zum Grad der Verbindlichkeit einer moralischen Handlung zuordnen. Blasi (1983) betont, dass die Verbindlichkeit der Urteile von der Selbstdefinition jeweiliger Personen abhängt. Eine moralische Person zu sein, erfordert in vielen Situationen, auf den eigenen Vorteil zu verzichten und zugunsten anderer zu handeln. Demnach würde ein Jugendlicher/eine Jugendliche nicht zum Täter/zur Täterin werden, wenn es ihm/ihr wichtig ist, eine moralische Person zu sein. Viktimisierung von Gleichaltrigen könnte nicht vollzogen werden, ohne die Selbstkonsistenz durch diese Handlungen zu gefährden.

Das Alter und das Geschlecht wurden als Kontrollvariablen mit aufgenommen. Die direkten Effekte des Geschlechts auf die aufgenommen Variablen entsprechen bisheriger Forschungsergebnisse zur Rolle des Geschlechts bei Callouss-unemotional Traits (Bleyer, Koglin \& Petermann, 2017; Fragkaki, Cima \& Meesters, 2016; Koglin \& Petermann, 2012) zu Gunsten der Mädchen, sowie dem vorhandenen Geschlechterunterschied in der moralischen Identität (Hardy, Walker, Olsen, Woodbury \& Hickman, 2014; Patrick, Rote, Gibbs \& Basinger, 2019). Anders als der von Patrick et al. (2019) herausgestellter Unterschied in der moralischen Identität zu Gunsten der Jungen zeigen sich die Unterschiede des Geschlechts in der moralischen Identität bei vorliegender und vergleich- 
barer Stichprobe jedoch zu Gunsten der Mädchen. Der Einfluss des Geschlechts auf Bullying widerspricht bisheriger Forschungsergebnisse, die von geschlechterunabhängigen Täterschaften berichten (Marées \& Petermann, 2009). Das Alter zeigt eine schwache aber signifikante Beziehung zu allen im Modell aufgenommenen Variablen. Die Ausprägungen der CU-Traits und Bullying steigen bei vorliegender Studie mit dem Alter an, die Ausprägung der moralischen Identität nimmt ab. Der Einfluss des Alters auf die moralische Identität ist sehr gering; auch in anderen Studien werden lediglich kleine Effekte berichtet (Hardy, Bean \& Olsen, 2015; Hardy et al., 2014; Weller \& Lagattuta, 2014). Insgesamt ist die Studienlage zum $\mathrm{Zu}$ sammenhang zwischen Alter und moralischer Identität nicht konsistent. Krettenauer, Murua und Jia (2016) sowie Krettenauer und Victor (2017) berichten von einem positiven Zusammenhang zwischen Alter und moralischer Identität, während andere Studien keinen Zusammenhang berichten (Hardy, 2006; Hardy et al., 2014; Krettenauer, 2011).

\section{Limitationen und Ausblick}

Wenngleich die präsentierten Auswertungen verschiedene Stärken aufweisen, sind zuletzt auch Einschränkungen dieser Studie anzumerken. Das querschnittliche Design dieser Studie erlaubt keinen Rückschluss auf die Entwicklung der CU-Traits, der moralischen Identität sowie auf das Bullying der Jugendlichen. Es ergab zudem ausschließlich die Möglichkeit, nach Täterschaften im letzten Schulhalbjahr zu fragen. Ein längerer Beobachtungszeitraum sollte in zukünftigen Untersuchungen berücksichtigt werden. Darüber hinaus ist selbstverständlich, dass die hier präsentierten Ergebnisse aufgrund der vorliegenden Gelegenheitsstichprobe nicht auf eine Grundgesamtheit generalisiert werden können. Weiterhin beruhen die Ergebnisse auf der Selbstbeurteilung der Jugendlichen. Wenngleich es in diesem Zusammenhang ein wichtiges Forschungsanliegen sein sollte, Geschlechts- als auch Alterseffekte differenziert herauszuarbeiten, war es innerhalb dieser Studie nicht das Ziel, diese Wechselwirkungen genauer zu analysieren, dies sollte künftig ausführlichere Berücksichtigung finden. Die Varianzaufklärung im Modell liegt bei $28 \%$. Es sollten in zukünftigen Modellen weitere entwicklungspsychologische Einflussfaktoren aufgenommen werden. Die Literatur lässt vermuten, dass es von hoher Relevanz ist, unter anderem die Kontextabhängigkeit aufgenommener Variablen dieser Studie mit zu berücksichtigen (Krettenauer \& Hertz, 2015; Oser, 2013; Pozzoli \& Gini, 2010; Waller \& Hyde, 2017).

\section{Implikationen und Schlussfolgerung}

CU-Traits und die moralische Identität konnten in dieser Studie als Einflussfaktoren auf Bullying identifiziert werden. Es liegen bereits verschiedene Präventionsprogramme vor, die Bullying an Schulen erfolgreich reduzieren können, wie. z.B. das Bullying Prevention Program von Olweus (1993) oder aus dem deutschsprachigen Raum "Mutig gegen Mobbing“ (Alsaker, 2017). Die vorliegenden Ergebnisse legen nahe zu überprüfen, ob in solchen Programmen gezielte Interventionen zur Förderung der moralischen Identität aufgenommen werden sollten. Hardy (2006) schlägt vor, das moralische Verhalten Jugendlicher wirksamer zu fördern, indem ein Schwerpunkt auf die Entwicklung der moralischen Identität gelegt wird. Hardy (2006) betont, dass Programme daran ansetzen sollten, Einzelpersonen bei der Entwicklung einer moralischen Identität fundiert zu unterstützen. Auch die hier durchgeführte Analyse identifizierte die moralische Identität als Prädiktor auf das Verhalten von Jugendlichen. Die Zusammenhänge zwischen CU-Traits und Bullying bestätigen Ergebnisse, aus denen abgeleitet werden kann, dass eine Förderung der sozial-emotionalen Kompetenzen zur Prävention von Bullying eingesetzt werden kann (Scheithauer \& Bull, 2007). Im Rahmen der vorliegenden Studie wurde eine Gelegenheitsstichprobe von Jugendlichen untersucht. Hohe Ausprägungen von CU-Traits, wie sie im Zusammenhang mit einer Störung des Sozialverhaltens vorkommen können, bestehen hier nicht. Die Items erfassen aber emotionale Kompetenzen wie Empathie und Emotionsausdruck (z. B. „Anderen fällt es leicht zu sagen, wie ich mich fühle“, „Ich verstecke meine Gefühle vor anderen"). Zur Reduktion von CU-Traits müssten hingegen gezielt sehr früh einsetzende Interventionen zur Förderung der sozial-emotionalen Entwicklung zur Anwendung kommen (Wilkinson, Waller \& Viding, 2016).

Die Ergebnisse zeigen für den deutschsprachigen Raum das Zusammenspiel zwischen CU-Traits, der moralischen Identität und Tätererfahrungen im Bullying. Ferner deuten die Befunde an, dass die moralische Identität und die damit einhergehende moralische Entwicklung wichtige Prädiktoren auf das Verhalten von Jugendlichen sind.

\section{Literatur}

Alsaker, F.D. (2017). Mutig gegen Mobbing: in Kindergarten und Schule. Bern: Hogrefe.

American Psychiatric Association (2013). Announcement. DSM-5. Retrieved from www.dsm5.org

Arbuckle, J. L. (2012). AmosTM 21.0 user's guide. Chicago: SPSS. 
Aquino, K. \& Reed, A. (2002). Self- importance of moral identity scale. Psyctests, 83, 10-11. https://doi.org/10.1037/t08774000

Baltes-Götz, B. (2008). Behandlung fehlender Werte in SPSS und Amos. Online-Dokumentation: http://www.uni-trier.de/index. php?id=23239

Bergmann, M. C., Baier, D., Rehbein, F. \& Mößle, T. (2017). Jugendliche in Niedersachsen. Ergebnisse des Niedersachsensurveys 2013 und 2015. (KFN-Forschungsberichte No. 131). Hannover: KFN.

Blasi, A. (1983). Moral cognition and moral action: A theoretical perspective. Developmental Review, 3, 178-210. https://doi. org/10.1016/0273-2297(83)90029-1

Bleyer, N., Koglin, U. \& Petermann, F. (2017). Callous-unemotional Traits und aggressives Verhalten. Zur Bedeutung der Bindungsqualität zu Eltern und Gleichaltrigen. Zeitschrift für Psychiatrie, Psychologie und Psychotherapie, 65, 175-185. https://doi.org/10.1024/1661-4747/a000318

Browne, K. A. \& Cudeck, J. S. (1993). Alternative ways of assessing equation model fit. In K. A. Bollen \& J. S. Long (Eds.), Testing structural equation models (pp. 136 -162). Newbury Park: Sage.

Cardinale, E. \& Marsh, A. (2020). The reliability and validity of the Inventory of Callous Unemotional Traits: A meta-analytic review. Assessment, 27, 57-71. https://doi.org/10.1177/ 1073191117747392

Ciucci, E., Baroncelli, A., Franchi, M, Golmaryami, F. \& Frick, P. (2014). The association between callous-unemotional traits and behavioral and academic adjustment in children: Further validation of the Inventory of Callous-Unemotional Traits. Journal of Psychopathology and Behavioral Assessment, 36, 189-200. https://doi.org/10.1007/s10862-013-9384-z

Craig, W., Harel-Fish, Y., Fogel-Grinvald, H., Dostaler, S., Hetland, J., Simons-Morton, B. et al. (2009). A cross-national profile of bullying and victimization among adolescents in 40 countries. International Journal of Public Health, 54, 216-224.

Doering, B. (2013). The development of moral identity and moral motivation in childhood and adolescence. In K. Heinrichs, F. Oser \& T. Lovat (Eds.), Handbook of moral motivation: Theories, models, applications (pp. 289 -306). Rotterdam: Sense Publishers. https://doi.org/10.1007/978-94-6209-275-4_17

Essau, C. A., Sasagawa, S. \& Frick, P. J. (2006). Callous-unemotional traits in community sample of adolescents. Assessment, 13, 454-469. https://doi.org/10.1177/1073191106287354

Fanti, K. A., Frick, P. J. \& Georgiou, S. J. (2009). Linking callousunemotional traits to instrumental and non-instrumental forms of aggression. Journal of Psychopathology and Behavioral Assessment, 31, 285-298.

Fragkaki, I., Cima, M. \& Meesters, C. (2016). The association between callous-unemotional traits, externalizing problems, and gender in predicting cognitive and affective morality judgments in adolescence. Journal of Youth and Adolescence, 45, $1917-$ 1930. https://doi.org/10.1007/s10964-016-0527-x

Frick, P. J. \& Hare, R. (2001). Antisocial process screening device. Toronto, Canada: Multi-Health Systems. https://doi.org/10. 1177/1073191103252347

Frick, P. J. (2004). The Inventory of Callous-Unemotional Traits. Unpublished rating scale. New Orleans, LA: University of New Orleans.

Frick, P. J. \& Salekin, R. T. (2005). Psychopathy in children and adolescents: The need for a developmental perspective. Journal of Abnormal Child Psychology, 33, 403 - 409. https://doi.org/10. 1007/s10802-005-5722-2

Gini, G., Pozzoli, T. \& Bussey, K. (2015). The role of individual and collective moral disengagement in peer aggression and bystanding: A multilevel analysis. Journal of Abnormal Child Psy- chology, 43, 441-452. https://doi.org/10.1007/s10802-0149920-7

Grundherr, M. Geisler, A. Stoiber, M. \& Schäfer, M. (2017). School bullying and moral reasoning competence. Social Development, 33, 278-294. https://doi.org/10.1111/sode.12199

Hardy, S. A. \& Carlo, G. (2005). Identity as a source of moral motivation. Human Development, 48, 232 - 256. https://doi.org/10. 1159/000086859

Hardy, S. A. (2006). Identity, reasoning, and emotion: An empirical comparison of three sources of moral motivation. Motivation and Emotion, 30, 207-215. https://doi.org/10.1007/s11031006-9034-9

Hardy, S., Bean, D. \& Olsen, J. (2015). Moral identity and adolescent prosocial and antisocial behaviors: Interactions with moral disengagement and self- regulation. Youth Adolescence, 44, 1542 - 1554. https://doi.org/10.1007/s10964-014-0172-1

Hardy, S. \& Carlo, G. (2011). Moral identity: what is it, how does it develop, and is it linked to moral action? Child Development Perspectives, 5, 212-218. https://doi.org/10.1111/j.17508606.2011.00189.x

Hardy, S. A., Walker, L. J., Olsen, J. A., Woodbury, R. D. \& Hickman, J. R. (2014). Moral identity as moral ideal self: Links to adolescent outcomes. Developmental Psychology, 50, 45-57. https:// doi.org/10.1037/a0033598

Hare, R. D. (1999). Without conscience: The disturbing world of the psychopaths among us. New York, NY: Guilford Press.

Hare, R. D. (2003). Hare Psychopathy Checklist-Revised manual (2nd ed.). Toronto: Multi-Health Systems.

Hart, D. (2005). The development of moral identity. In G. Carlo \& C. P. Edwards (Eds.), Nebraska symposium on motivation: Moral development through the lifespan: Theory, research, and application (Vol. 51, pp. 165-196). Lincoln: University of Nebraska Press.

Hart, D., Atkins, R. \& Ford, D. (1998). Urban america as a context for the development of moral identity in adolescence. Journal of Social Issues, 54, 513-530. https://doi.org/10.1111/00224537.801998080

Homburg, C. \& Baumgartner, H. (1995). Beurteilung von Kausalmodellen. Marketing ZFP, 17, 162-176.

Homburg, C. \& Giering, A. (1996). Konzeptualisierung und Operationalisierung komplexer Konstrukte- Ein Leitfaden für die Marketingforschung. Marketing: Zeitschrift für Forschung und Praxis, 18, 5-24.

Hymel, S., Rocke Henderson, N. \& Bonanno, R. A. (2005). Moral disengagement: A framework for understanding bullying among adolescents. Journal of Social Sciences, 8, 1-11.

Jantzer, V., Haffner, J., Parzer, P. \& Resch, F. (2012). Opfer von Bullying in der Schule. Depressivität, Suizidalität und selbstverletzendes Verhalten bei deutschen Jugendlichen. Kindheit und Entwicklung, 21, 40-46. https://doi.org/10.1026/09425403/a000068

Klomek, A. B., Barzilay, S., Apter, A., Carli, V., Hoven, C. W., Sarchiapone, M. et al. (2019). Bi-directional longitudinal associations between different types of bullying victimization, suicide ideation/attempts, and depression among a large sample of european adolescents. Journal of child Psychology and Psychiatry, 60, 209-215. https://doi.org/10.1111/jcpp.12951

Koglin, U. \& Daseking, M. (2017). Fragebogen zur Moralentwicklung im Jugendalter. (Unveröffentlichte Publikation). Carl von Ossietzky Universität, Oldenburg.

Koglin, U. \& Petermann, F. (2007). Psychopathie im Kindesalter. Kindheit und Entwicklung, 16, 250-266. https://doi.org/10. 1026/0942-5403.16.4.260

Koglin, U. \& Petermann, F. (2012). Callous- unemotional traits: Verhaltensprobleme und prosoziales Verhalten bei Kindergar- 
tenkindern. Kindheit und Entwicklung, 21, 141-150. https:// doi.org/10.1026/0942-5403/a000080

Krettenauer, T. (2011). The dual moral self: Moral centrality and internal moral motivation. The Journal of Genetic Psychology, 172, $\quad 309-328 . \quad$ https://doi.org/10.1080/00221325.2010. 538451

Krettenauer T. (2013) Moral motivation, responsibility and the development of the moral self. In K. Heinrichs, F. Oser \& T. Lovat (Eds.), Handbook of moral motivation: Theories, models, applications (pp. 215 -228). Rotterdam: Sense Publishers. https:// doi.org/10.1007/978-94-6209-275-4_13

Krettenauer, T. \& Hertz, S. (2015). What develops in moral identities? A critical review. Human Development, 58, 137-153. https://doi.org/10.1159/000433502

Krettenauer, T., Murua, L. A. \& Jia, F. (2016). Age-related differences in moral identity across adulthood. Developmental Psychology, 52, 972 - 984. https://doi.org/10.1037/dev0000127

Krettenauer, T. \& Victor, R. (2017). Why be moral? Moral identity motivation and age. Developmental Psychology, 53, $1589-$ 1596. https://doi.org/10.1037/dev0000353

Kurek, A., Jose, P. \& Stuart, J. (2019). 'I did it for the LULZ': How the dark personality predicts online disinhibition and aggressive online behavior in adolescence. Computers in Human Behavior, 98, 31 - 40. https://doi.org/10.1016/j.chb.2019.03.027

Little, R. J. A. (1988). A test of missing completely at random for multivariate data with missing values. Journal of the American Statistical Association, 83, 1198-1202.

Malti, T., Gummerum, M., Keller, M. \& Buchmann, M. (2009). Children's moral motivation, sympathy, and prosocial behavior. Child Development, 80, 442-460. https://doi.org/10.1111/j. 1467-8624.2009.01271.x

Malti, T. \& Krettenauer, T. (2013). The relation of moral emotion attributions to prosocial and antisocial behavior: A meta-analysis. Child Development, 84, 397-412. https://doi.org/10. 2307/23469337

Marées, N. \& Petermann, F. (2009). Bullying an Grundschulen. Formen, Geschlechtsunterschiede und psychosoziale Korrelate. Psychologische Rundschau, 60, 152-162. https://doi.org/ 10.1026/0033-3042.60.3.152

Munoz, L. C., Qualter, P., Padgett, G. (2011) Empathy and bullying: Exploring the influence of callous-unemotional traits. Child Psychiarty and Human Development, 42, 183-96. https://doi. org/10.1007/s10578-010-0206-1

Muratori, P., Paciello, M., Buonanno, C., Milone, A., Ruglioni, L., Lochman, J. E. \& Masi, G. (2017). Moral disengagement and callous-unemotional traits: A longitudinal study of Italian adolescents with a disruptive behaviour disorder. Criminal Behaviour and Mental Health, 27, 514-524. https://doi.org/10.1002/ cbm.2019

Olweus, D. (1993). Bullying at school: What we know and what we can do. Oxford: Blackwell Publishers. https://doi.org/10.1002/ pits. 10114

Olweus, D. (2013). School bullying: Development and some important challenges. Annual Review of Clinical Psychology, 9, 751 780. https://doi.org/10.1146/annurev-clinpsy-050212-185516

Oser, F. (2013) Models of moral motivation. In K. Heinrichs, F. Oser \& T. Lovat (Eds.), Handbook of moral motivation: Theories, models, applications (pp. 289 -306). Rotterdam: Sense Publishers. https://doi.org/10.1007/978-94-6209-275-4_2

Patrick, R. B., Rote, W. M., Gibbs, J. C. \& Basinger, K. S. (2019). Defend, stand by, or join in? The relative influence of moral identity, moral judgment, and social self-efficacy on adolescents' bystander behaviors in bullying situations. Journal of
Youth and Adolescence, 48, 2051 -2064. https://doi.org/10. 1007/s10964-019-01089-w

Petermann, F. \& Koglin, U. (2013). Aggression und Gewalt von Kindern und Jugendliche: Hintergründe und Praxis. Berlin: Springer.

Pozzoli, T. \& Gini, G. (2010). Active defending and passive bystanding behavior in bullying: The role of personal characteristics and perceived peer pressure. Journal of Abnormal Child Psychology, 38, 815-827. https://doi.org/10.1007/s10802-0109399-9

Scheithauer, H. \& Bull, H. (2007). Unterrichtsbegleitende Förderung sozialer Kompetenzen und Prävention von Bullying im Jugendalter: das fairplayer.manual. Gruppenpsychotherapie und Gruppendynamik, 43, 277 -293. https://doi.org/10.13109/grup. 2007.43.4.277

Scheithauer, H., Hayer, T. \& Petermann, F. (2003). Bullying unter Schülern. Erscheinungsformen, Risikobedingungen und Interventionskonzepte. Göttingen: Hogrefe.

Statistisches Bundesamt (Hrsg.). (2013). Bevölkerung und Erwerbstätigkeit - Bevölkerung mit Migrationshintergrund. (Fachserie 1, Reihe 2.2). Wiesbaden: Statistisches Bundesamt.

Thornberg, R. \& Jungert, T. (2017). Callous-unemotional traits, harm-effect moral reasoning, and bullying among swedish children. Child Youth Care Forum, 46, 559 - 575. https://doi.org/ 10.1007/s10566-017-9395-0

Waller, R. \& Hyde, L. (2017). Callous-unemotional behaviors in early childhood: Measurement, meaning, and the influence of parenting. Child Development Perspectives, 11, 120-126.

Wang, C. X., Ryoo, J. H., Swearer, S. M., Turner, R. \& Goldberg, T. S. (2017). Longitudinal relationships between bullying and moral disengagement among adolescents. Journal of Youth and Adolescence, 46, 1304-1317. https://doi.org/10.1007/s10964016-0577-0

Weller, D. \& Lagattuta, K. H. (2014). Children's judgments about prosocial decisions and emotions: Gender of the helper and recipient matters. Child Development, 85, 2011-2028. https:// doi.org/10.1111/cdev.12238

Wilkinson, S., Waller, R. \& Viding, E. (2016). Practitioner Review: Involving young people with callous unemotional traits in treatment-does it work? A systematic review. Journal of Child Psychology and Psychiatry, 57, 552 -565. https://doi.org/10.1111/ jcpp. 12494

Wright, M. F., Harper, B. D. \& Wachs, S. (2019). The associations between cyberbullying and callous-unemotional traits among adolescents: The moderating effect of online disinhbition. Personality and Individual Differences, 140, 41 - 45. https://doi.org/ 10.1016/j.paid.2018.04.001

Zych, I., Ttofi, M. M. \& Farrington, D.P. (2019). Empathy and callous-unemotional traits in different bullying roles: A systematic review and meta-analysis. Trauma, Violoence \& Abuse, 20, 3-21. https://doi.org/10.1177/1524838016683456

\section{Förderung}

Open Access-Veröffentlichung ermöglicht durch die Carl von Ossietzky Universität Oldenburg.

\section{Neele Schipper, M. Ed.}

Carl von Ossietzky Universität Oldenburg

Fachbereich Psychologie in der

Sonder- und Rehabilitationspädagogik

Ammerländer Heerstraße 114-118

26129 Oldenburg

neele.schipper@uni-oldenburg.de 